\title{
Integração das tecnologias digitais para exploração e desenvolvimento do raciocínio visuoespacial na aprendizagem de geometria.
}

\author{
Claudiomir Feustler Rodrigues de Siqueira* - IFRS / PPGIE - UFRGS - \\ claudiomir.siqueira@canoas.ifrs.edu.br \\ Jaqueline Molon* - IFRS / PPGIE - UFRGS - jaqueline.molon@canoas.ifrs.edu.br \\ Sérgio Roberto Kieling Franco - PPGIE/PPGEdu - UFRGS - sergio.franco@ufrgs.br
}

Resumo: O presente estudo investigou a utilização e exploração do raciocínio visuoespacial, aliado à integração de tecnologias educacionais, para o favorecimento do ensino e aprendizagem de geometria. A partir de uma proposta didática, com a inserção de jogos digitais no meio educacional, foram exploradas diferentes situações de aprendizagem onde os conceitos básicos de geometria euclidiana foram trabalhados com licenciandos em matemática. $\mathrm{Na}$ análise dos resultados, constatou-se que aqueles que conseguiram melhor desempenho, apresentavam habilidade visuoespacial mais apurada do que os demais. Dessa forma, focando nos processos cognitivos além dos aspectos didáticos-pedagógicos, com a integração tecnológica, promoveu-se junto aos futuros docentes o potencial que o desenvolvimento do raciocínio visuoespacial pode propiciar.

Palavras-chave: raciocínio visuoespacial; tecnologias digitais; geometria; ensino e aprendizagem.

\section{Integration of digital technologies for the visuospatial reasoning exploration and development in geometry learning}

Abstract: The present study investigated the use and exploration of visuospatial reasoning, combined with the integration of educational technologies, to favor geometry teaching and learning. From a didactic proposal, with the insertion of digital games in the educational environment, different learning situations were explored where the basic concepts of Euclidean geometry were worked with undergraduates in mathematics. In the results analysis, it was found that those who achieved better performance had more visuospatial ability than the others. Thus, focusing on cognitive processes beyond the didactic-pedagogical aspects, with the technological integration, it was promoted with the future teachers the potential that the visuospatial reasoning development can propitiate.

Keywords: visuospatial reasoning; digital technologies; geometry; teaching and learning.

\section{Introdução}

Em todas as áreas da matemática é necessário, muitas vezes, imaginar objetos estáticos ou dinâmicos e agir sobre eles mentalmente, projetando rotações, ampliações ou reduções, translações, deslocamentos, etc. Essa habilidade de visualizar, manipular, fazer operações mentais e saber interpretar esses resultados servirá de base para a compreensão do objeto em estudo. Consequentemente, a "habilidade visual nos ajuda entender, apreciar e interpretar nosso mundo geométrico" (NCTM, 2000 apud Lowrie et al., 2017, p.1). Nesse sentido, pesquisas têm buscado compreender, cada vez mais, como o raciocínio visuoespacial pode auxiliar no ensino e aprendizagem de matemática. Em particular, na área de geometria, pode ser citado o estudo de Sinclair et al., (2016), que traz um resumo sobre o tema, apresentando-o como uma tendência mundial de investigação na área que tem avançado significativamente, mas que ainda requer muitos estudos.

A articulação das habilidades visuoespaciais com o processo de ensino e aprendizagem e avaliação de matemática está promovendo melhorias na geometria escolar. Diversos estudos (Bruce; Hawes, 2015; Hallowell et al., 2015; Soury-Lavergne; 
Maschietto, 2015; Moss et al., 2015; Sinclair; Bruce, 2015) destacaram experiências de sucesso sobre raciocínio espacial e sua conexão com a matemática apoiadas pelas tecnologias educacionais na escola primária. Nesse contexto, Davis e o Grupo de Estudo Raciocínio Espacial (2015) publicaram um livro, no qual discutem a inserção de práticas que exploram o raciocínio espacial nas séries iniciais, propondo adaptações curriculares para sua contemplação como forma de melhorar o desempenho acadêmico. Nessa mesma direção, pesquisas apontaram para a forte relação entre o treinamento da capacidade visuoespacial e o desempenho matemático com estudantes do ensino fundamental (Lowrie et al., 2017) e do ensino médio (Ramful et al., 2017).

Dada a sua importância essa é uma temática que ganha força em todas as áreas de ensino, ainda mais com os avanços tecnológicos e com a possibilidade de intervenções em ambientes dinâmicos. Não ficando restrita à matemática, essa habilidade é relevante para outras áreas das Ciências, Tecnologia, Engenharia e Matemática (STEM) (Wai et al., 2009; Utta et al., 2013). Tal capacidade refletiu na escolha de carreiras científicas conforme destaca Wai et al. (2009). Em um estudo longitudinal, comparando testes da adolescência com dados contemporâneos, estes autores constataram que aqueles que tinham bem essa habilidade desenvolvida atingiram formação e ocupações mais altas em STEM. Devido a esses resultados os pesquisadores sugerem avaliar a capacidade visuoespacial como uma forma de caça-talentos com potencial para STEM, pois ela desempenha um papel crítico para a especialização profissional.

Nesse contexto, são apresentadas diferentes situações de ensino e aprendizagem de geometria, realizadas com licenciandos em matemática, contemplando a utilização e exploração do raciocínio visuoespacial com a integração de tecnologias digitais. Para tanto, transita-se num cenário de aprendizagem pautado inicialmente sob aspectos lúdicos, mas que em determinado momento exigiu maior rigor matemático, com o objetivo de identificar potencialidades do raciocínio visuoespacial de cada um dos estudantes durante as atividades e auxiliá-los no seu desenvolvimento cognitivo, bem como destacar a relevância do tema para os mesmos enquanto futuros docentes.

\section{Formação docente: TDICs e a Geometria}

A formação docente está cada vez mais atrelada a uma prática profissional múltipla, exigindo-se cursos ainda mais completos e concisos com às demandas da sociedade. Nessa direção, as Diretrizes Curriculares Nacionais para a Formação Docente, tanto inicial quanto continuada, deixam em evidência, em seu artigo $5^{\circ}$, que o percurso formativo desses cursos deve, entre outros aspectos, conduzir os egressos à integração competente das Tecnologias Digitais de Informação e Comunicação (TDICs) "para o aprimoramento da prática pedagógica e a ampliação da formação cultural dos(das) professores(as) e estudantes" (Brasil, 2015, p.6).

Nesse sentido, as instituições formadoras vêm promovendo reformas curriculares que visam atender às exigências do cenário educacional do século XXI, bem como as necessidades evidenciadas, de modo específico, pelo rápido desenvolvimento tecnológico que não podem ser desconsiderados no campo da educação. Diante disso, algumas instituições têm passado a considerar, em seus currículos, as TDICs não apenas contempladas em disciplinas específicas, mas imbricadas em diversas disciplinas nas quais os objetivos voltam-se ao "pensar-com", através da exploração de possibilidades ofertadas pelas tecnologias.

\footnotetext{
${ }^{1}$ Pensar-com é destacado por Basso e Notare (2015) como conceito que significa que a utilização dos recursos digitais servirá para favorecer o pensar em matemática, desencadeando "modos criativos de resolver problemas em e com matemática" (p.10).
} 
Dessa forma, a formação potencializa a exploração de possibilidades dos recursos tecnológicos para serem integrados no processo de ensino, aprendizagem e avaliação, pelos futuros profissionais, principalmente quando estes passarem a atuar na educação básica. Consequentemente, o posicionamento crítico, autoconfiante, frente às TDICs advirá de diferentes vivências teóricas e práticas nos ambientes formativos, que buscam fortalecer os saberes relacionados às tecnologias.

Nas aulas de matemática do ensino básico, a geometria muitas vezes é deixada de lado ou fica para um segundo plano. Resultado desse descaso é identificado no ensino superior quando os alunos evidenciam muitas dificuldades de aprendizagem apresentando "pouca compreensão dos objetos geométricos, confundindo propriedades do desenho com propriedades do objeto" (Gravina, 1996, p.1). Talvez o importante, nesse momento que apresenta grande demanda de formação de professores e, diante das dificuldades destacadas, seja tentar esclarecer que habilidades e competências geométricas são necessários nos dias de hoje. Essa discussão servirá como suporte para a tentativa de apresentar uma resposta ao seguinte questionamento/dúvida: "Por que ensinar ou aprender geometria?".

Documentos padrões sobre o ensino de matemática e formação de professores produzidos pela National Council of Teachers of Mathematics - NCTM (2000) destacam que o domínio da construção de modelos geométricos e do pensamento espacial são ferramentas importantes para a resolução de problemas, sejam eles geométricos ou de outras áreas, tanto da matemática quanto das ciências em geral. Na mesma direção, os Parâmetros Nacionais Curriculares (PCNs) ressaltam a importância da aprendizagem de geometria no ensino básico por possibilitar os "primeiros contatos com a necessidade e as exigências estabelecidas por um raciocínio dedutivo" (Brasil, 1998, p.86), sendo "campo fértil de situações-problema que favorece o desenvolvimento da capacidade para argumentar e construir demonstrações" (Brasil, 1998, p.122). Portanto, sua aprendizagem favorece "desenvolver um tipo de pensamento particular para compreender, descrever e representar, de forma organizada, o mundo em que vive" (Brasil, 1998, p.122).

Cabe salientar ainda que tanto a Base Nacional Comum Curricular para o Ensino Fundamental, recentemente aprovada, quanto a do Ensino Médio, atualmente em discussão, apontam que a "Geometria envolve o estudo de um amplo conjunto de conceitos e procedimentos necessários para resolver problemas do mundo físico e de diferentes áreas do conhecimento" (Brasil, 2018a, p.269) e destacam o papel do pensamento geométrico "necessário para investigar propriedades, fazer conjecturas e produzir argumentos geométricos convincentes" (p.271). Estes documentos chamam atenção, inclusive, para a necessidade da utilização da geometria dinâmica e do estudo da geometria de forma integrada com as demais unidades temáticas (álgebra, grandezas e medidas, números e probabilidade e estatística (Brasil, 2018a, 2018b).

A palavra geometria traz consigo uma multiplicidade de significados de interesse matemático, que cada vez são mais importantes para a sociedade, destacando-se pela amplitude e versatilidade de suas aplicações. Nesse sentido, Alsina, Fortuny e Pérez (1997) realçam a relevância de contemplar os futuros cidadãos com uma cultura geométrica, o que requer desenvolvimento de habilidades específicas, com vocabulário adequado e com uma visão global das aplicações atuais. Para atingir esse patamar é preciso motivar e habilitar o futuro docente a trabalhar com algo que, muitas vezes, lhe foi negligenciado. Para isso, é necessário promover uma formação que apresente os campos de aplicação da área. Considerando os avanços tecnológicos pode-se utilizar de exemplos voltados para a precisão geométrica necessária à realidade virtual aumentada, aos ambientes virtuais de aprendizagem e à computação gráfica, entre outros. 


\section{Descrição da proposta e dos recursos tecnológicos}

O estudo foi realizado a partir da coleta de dados de duas experiências desenvolvidas com alunos da Licenciatura em Matemática do IFRS - Campus Canoas, matriculados na disciplina de "Tecnologias no Ensino de Matemática" e de "Geometria I", que são ofertadas regularmente para o $3^{\circ}$ semestre do curso. O trabalho foi realizado em dois encontros de $4 \mathrm{~h}$ cada. Num primeiro momento, foi criada uma atividade dentro da plataforma Gartic, para explorar conhecimentos matemáticos prévios sobre geometria euclidiana. E em outro, foi utilizado o aplicativo Euclidea, a fim de revisar conceitos e construções euclidianas possíveis com régua e compasso num ambiente digital e com rigor matemático.

O Gartic é uma plataforma online multiplayer com foco em jogos de desenhos, onde o objetivo é acumular o maior número de pontos obtidos ao acertar mais rapidamente o que o outro está desenhando ou por interpretações corretas do seu desenho. A cada rodada um dos jogadores desenha/constrói uma imagem sem utilizar letras e símbolos, associada a um determinado item, palavra ou frase da sala tema, e os demais tentam acertar/deduzir. Quanto maior o número de acertos que o desenho proporciona mais pontos o desenhista acumula. "Caso alguém acerte esta palavra, tanto ela, quanto o desenhista ganham pontos e a cada novo acerto a premiação de pontos passa a ser menor, tendo o limite mínimo de 1 ponto" (Gartic, 2016, p.1).

Figura 1 - Área de desenho do Gartic

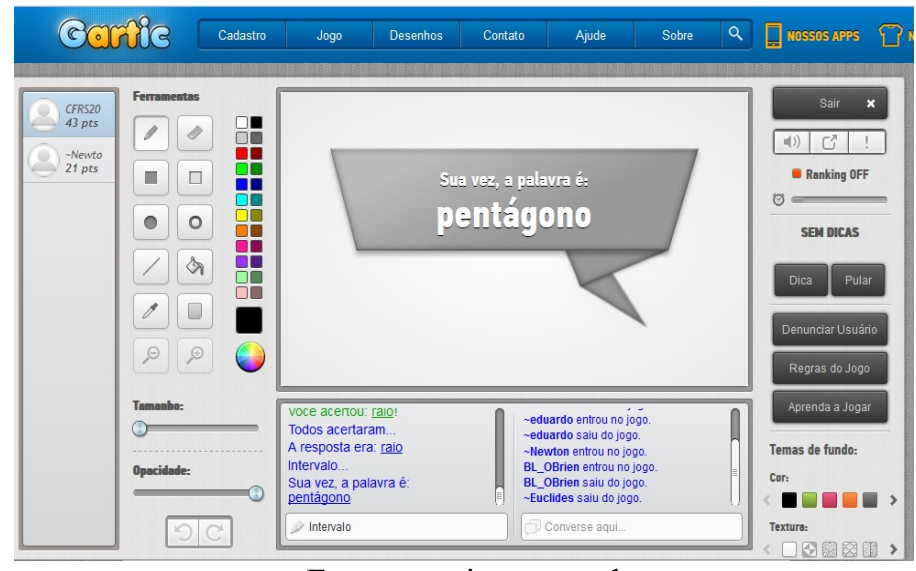

Fonte: arquivo pessoal

Os usuários ou jogadores podem utilizar as salas/temas existentes ou criar conforme seu interesse. Por exemplo, nessa experiência criou-se o tema geometria, com digitação de 60 termos/conceitos vinculados, estabelecendo graus de dificuldade (fácil, médio ou difícil) associados a cada construção/esboço na forma de desenho do conceito ou ao entendimento/interpretação desse conceito/figura. Exemplificando, o termo "retângulo" foi considerado "fácil" e o termo "baricentro" foi classificado como "médio". Durante o jogo, de forma cíclica, todos passavam pela função de desenhador. Enquanto um desenhava os outros apresentavam respostas e, caso estas não estivessem corretas, ficavam visíveis a todos os jogadores num chat. Errar não penalizava e quem acertasse a ferramenta destacava seu nickname de jogador nesse espaço. $\mathrm{O}$ ambiente de desenho assemelha-se com o Paint, conforme se pode observar na figura 1, acima.

O Euclidea é um recurso didático digital que explora construções geométricas euclidianas possíveis a partir do uso de régua e compasso. Pode ser descrito como um puzzle com ambiente de geometria dinâmica que apresenta desafios e orientações, desde noções básicas, de um nível bem inicial até mais avançado, ao longo de mais de 120 desafios distribuídos em 15 níveis. Ele é gratuito quando você utiliza a versão desktop 
(http://www.euclidea.xyz/) e, enquanto ir resolvendo todas as construções de forma otimizada, com o menor número de movimentos (elementos). Em versões pagas disponíveis para $i O S$ ou Android é possibilitado "pular desafios" (Euclidea, 2018) o que pode ser uma estratégia viável caso o estudante não queira ficar muito tempo em uma tarefa específica ou tiver dificuldades, exemplificando. Na figura 2 está em destaque a construção de um pentágono regular inscrito numa circunferência com esse recurso.

Figura 2 - Exemplo da solução de um desafio

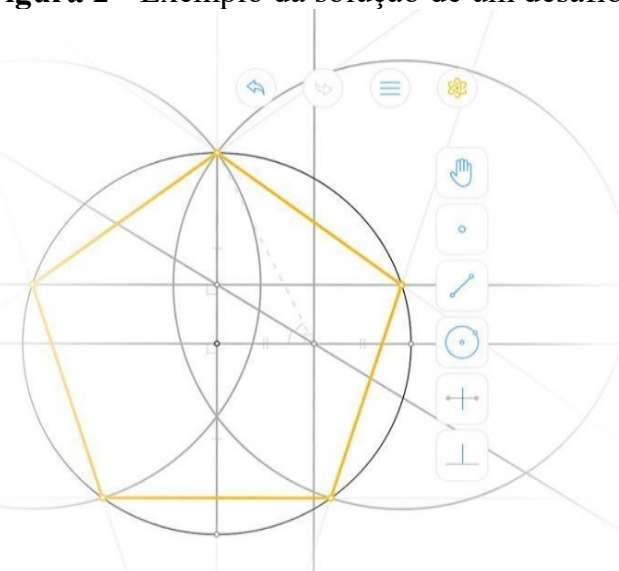

Fonte: https://www.euclidea.xyz/images/euclidea/IntroBg.jpg

Inserindo-se numa proposta de jogos voltados ao desenvolvimento do pensamento e ao estímulo ao raciocínio dedutivo e encadeamento lógico de ações, elementos da geometria euclidiana ganham robustez. A partir de pontos, retas, circunferências e construções elementares de geometria euclidiana, novos desafios são lançados. Ao final de cada desafio, a construção "solução" provando que ela satisfaz a declaração do problema deve ser "dinâmica". Não é suficiente apenas coincidir visualmente com a resposta correta, deve garantir a possibilidade de movimento sem colapsar, o que permite explorar ainda mais o objeto/propriedade com a ferramenta "mover", do mesmo modo que construções dinâmicas realizadas em softwares como o Geogebra.

\section{Análise e discussão dos dados}

A experiência com o uso da plataforma de desenhos Gartic teve a participação de 11 estudantes, identificados ao longo do texto por A1, A2, ... e A11. Foram feitas filmagens para a coleta de dados. A proposta inseriu-se na retomada de conceitos iniciais de geometria euclidiana, servindo como diagnóstico inicial da aprendizagem da turma sobre esses conteúdos, conforme os recortes que serão apresentados a seguir.

Na figura 3 uma aluna, na sua vez, tinha a tarefa de desenhar uma esfera, para os demais "jogadores" ela desenhou um círculo. Na rodada seguinte, um colega sugeriu outra possibilidade de desenho. Observa-se que a aluna A1 ficou alguns segundos pensando o que fazer e como fazer. Sabia o que é uma esfera, no entanto, não conseguiu imaginar uma boa forma de representá-la no plano. Para o aluno A8, estava bem claro como representá-la de forma que os demais entendessem sua informação.

Figura 3 - Desenho/representação de uma esfera
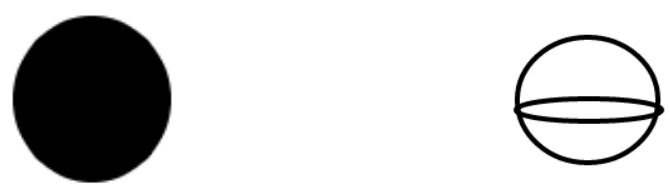

Desenho da aluna A1.

Sugestão do aluno A8.

Fonte: arquivo pessoal 
Na sequência (figura 4) são apresentados mais recortes dos desenhos feitos pelos alunos. No item (a) identifica-se a necessidade de elencar propriedades para definir um triângulo retângulo, o que evidencia um conhecimento mais aprofundado. A aluna A4, desenhista da rodada, julgou necessário construir um retângulo para a partir daí ressaltar o ângulo reto, mostrando certo rigor matemático em sua construção e maior exploração da habilidade visuoespacial, tendo em vista que seu primeiro esboço, com um triângulo qualquer aliado à tentativa de cruzar duas retas e obter retas perpendiculares, não lhe fornecia claramente o ângulo reto. A imagem (b) é uma tentativa de representar o termo "bissetriz". Nesse exemplo, o aluno A2 começou desenhando um "triângulo qualquer", após destacou um dos ângulos e desenhou um segmento de reta que partiu desse vértice. Foi possível constatar nas filmagens que ele estava prestes a "dividir o ângulo ao meio" e por um instante de dúvida desenhou, a mediana, pois tentou finalizar o segmento de reta no ponto médio do lado oposto, o que deixou em evidência o significado do termo atrelado visualmente a um triângulo, fato que o aluno A2 tomou como referência. Isso pode ser resultado da forma como professores e livros didáticos costumam apresentar o conteúdo.

O item (c) expressa a representação de um cone. A utilização de uma elipse, esboçando uma circunferência em perspectiva na base permitiu que todos pudessem interpretar, de forma correta, este desenho. No recorte (d) tem-se a representação do baricentro de um triângulo realizada por A2. Após desenhar o triângulo, suas medianas e marcar o ponto de encontro delas, o aluno achou necessário nomeá-lo por ponto "G". Esse fato traz evidências de que A2 vinculou a informação visuoespacialmente (Owens, 2014). Pode-se supor que o aluno memorizou esse fato a partir de uma aula de geometria, após observar a construção no Geogebra, que nomeou automaticamente os vértices (A, B, C) e os pontos médios de cada lado $(\mathrm{D}, \mathrm{E}, \mathrm{F})$, sendo o próximo ponto, intersecção das medianas, nomeado automaticamente como letra G.

Figura 4 - Recortes da tentativa de representar alguns termos geométricos

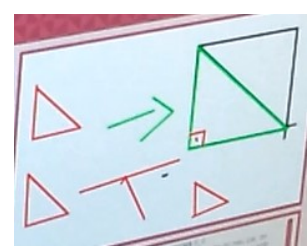

(a) - A4

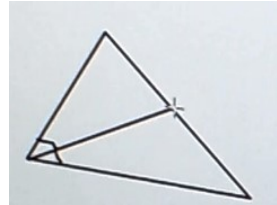

(b) - A2

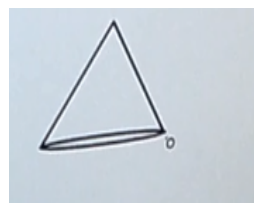

(c) - A10

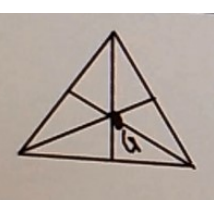

(d) - A2

Fonte: Arquivo pessoal

A reflexão sobre o entendimento de um conceito e o que é necessário apresentar para que outra pessoa compreenda o que está sendo construído, a fim de receber mais pontos por ter conseguido construir um "desenho" que permitiu aos colegas identificar qual conceito/objeto era proposto, possibilitou ao futuro profissional, também, refletir e aprender dentro da sua formação sobre sua própria prática no sentido atribuído por Schon (1997). Concomitante ao desenvolvimento da atividade, metáforas e/ou termos geométricos não formais foram utilizadas. Por exemplo, redondo para descrever círculo, circunferências, base de cone, etc. A ilustração de um conceito/termo sem o auxílio de palavras exigiu mais conhecimento do tema. Na busca de ser interpretado corretamente antecipações mentais dos termos eram feitas, para depois passar a realizar as construções. Ao mesmo tempo, outras soluções eram pensadas enquanto a construção era feita, o que nem sempre resultava em uma representação suficiente para ser interpretada conforme o proponente pretendia. As respostas fornecidas de forma simultânea à aparição dos traços 
e primeiro esboço do objeto que estava sendo construído, faziam os alunos percorrerem várias imagens mentalmente, adequando-as a cada novo estágio da construção que surgia, refutando e criando hipóteses para o que se aproximava, a figura que ganhava forma geométrica. Também eram levantadas hipóteses do que não se enquadrava no que estava sendo representado.

Durante as construções e interpretações dos desenhos três alunos (A2, A5, A9) recorriam frequentemente ao Google para buscar o significado "textual" do conceito ou para verificar a grafia correta. Exemplificando: o aluno A2 pesquisou o que era triângulo acutângulo, para então construir sua representação, o discente A5 buscou "tipos de triângulos" para tentar interpretar o triângulo que estava desenhado, enquanto que A9 já tinha feito interpretações visuais sobre triângulos, pois identificou que se tratava da classificação dos triângulos quanto à medida dos lados, apoiando-se da consulta ao site para relembrar a terminologia associada. Os termos "losango" e "isósceles" foram pesquisados para fins de grafia. No entanto, era uma busca que requer muita velocidade, pois era necessário responder algo o quanto antes.

A ferramenta apresenta limitações, principalmente no que se refere a unicidade de nomenclatura como resposta a determinada construção, que poderia admitir válidas expressões equivalentes. Contudo, a adaptabilidade dessa ferramenta aliada aos seus recursos de interatividade, permite ampliar sua utilização para o desenvolvimento de outras atividades além do ensino, aprendizagem e avaliação de Geometria. Por exemplo, poderão ser criados tópicos para auxiliar na alfabetização, no ensino de línguas, artes, geografia, sequências lógicas, etc. Com isso o olhar didático sobre as possibilidades de desenvolvimento do raciocínio visuoespacial pode favorecer o ensino e aprendizagem de forma interdisciplinar dentro de outros contextos e situações nas quais os sujeitos estão imersos/inseridos.

A proposta com o Euclidea contou com a participação de 16 estudantes. A coleta de dados foi feita a partir de registros fotográficos e de um questionário/feedback no Moodle acerca da utilização do aplicativo. Essa experiência exigia rigor matemático e capacidade visual mais apurada, uma vez que para realizar a prova de determinado conceito de forma dedutiva, na maioria das vezes, era preciso recorrer a determinada propriedade geométrica ou constatação verificada em alguma construção anterior.

A relevância das habilidades visuoespaciais ficaram evidentes no processo de resolução das construções. Exemplificando, essa capacidade auxiliou o aluno A1, que depois de certo tempo, conseguiu chegar na solução sozinho, conforme seu comentário: "num desafio que pedia para encontrar o ângulo de $60^{\circ}$, só encontrei a forma correta de se fazer quando percebi que poderia seguir a mesma ideia do desafio anterior, que seria fazer um triângulo equilátero, o qual por sua vez possuía três ângulos de $60^{\circ}$.

No entanto, o estudante A15, apresentou dificuldades no raciocínio espacial e não conseguiu solucionar o desafio sozinho: "logo na segunda tarefa foi necessário a professora falar sobre a utilização de um triângulo equilátero, assim ficou fácil fazer um ângulo de $60^{\circ}$ !'. No desafio anterior, foi proposto a construção de um triângulo equilátero, que foi feito com sucesso. Este aluno tem conhecimento de que todos os ângulos, desse tipo de triângulo, possuem mesma medida e de que a soma deles é $180^{\circ}$, ainda assim, não conseguiu transferir/agregar sozinho essas informações para obter a construção pedida, sem o auxílio da professora.

Conforme os feedbacks dos alunos, o aplicativo é muito motivador e os auxiliou na revisão de conteúdos de geometria. A maioria descreveu que provavelmente seguirá utilizando-o para estudar e todos destacaram que pretendem inserir em sua prática docente. O idioma na versão desktop apareceu como um limitador. Entretanto, se considerado sob aspectos interdisciplinares esse fato pode ser contornado e, além disso, 
conhecer outro idioma é uma necessidade na sociedade atual, ou seja, esse aspecto inicialmente considerado desfavorável pode se transformar em desafio na medida em que a interação com o aplicativo for intensificada.

A realização de construções em ambientes dessa natureza, portanto, possibilita aos estudantes refinar os processos de construção à medida que os desafios se tornam cada vez mais elaborados, ou seja, envolvendo um número maior de elementos a serem considerados, bem como de propriedades geométricas envolvidas (Laborde et al., 2006). Nesse contexto, o desenvolvimento do raciocínio visuoespacial progride no momento em que o sujeito se depara com determinada figura e precisa agir sobre, realizando novas ações, na busca de alternativas para construir a demonstração que satisfaz a solução do desafio, por exemplo.

Analogamente, em ambientes semelhantes ao Euclidea "os alunos aprendem coordenando e refletindo sobre a forma de suas interações" (Hoylos, 1995 apud Laborde, 2006, p.291, trad. nossa) e, assim, tanto os conceitos teóricos quanto os aspectos vinculados à visualização são potencializados. Dessa forma, as intervenções voltadas ao treinamento das habilidades espaciais proporcionam um ganho importante no desempenho matemático dos estudantes, o que deixa em evidência a necessidade de promover o desenvolvimento do raciocínio viosuoespacial, que pode se dar através de propostas como as que foram analisadas neste trabalho.

\section{Considerações finais}

Os currículos escolares (Lowrie, Diezmann, 2010) e as avaliações em larga escala (Kosslyn, 2006) estão se tornando cada vez mais espaciais, explorando informações matemáticas de natureza gráfica. Da mesma forma, está ocorrendo uma crescente exigência de proficiência da capacidade visuoespacial nas demais disciplinas do currículo (Lowrie et al. 2017). Assim intervenções para o desenvolvimento dessas habilidades se tornam profícuas. A partir das práticas descritas neste estudo, os licenciandos foram expostos a situações que exigiam a realização de ações coordenadas com foco na construção ou identificação de determinados elementos geométricos.

Propostas dessa natureza transcendem os conteúdos específicos, uma vez que para realizar as atividades é necessário, também, demonstrar conhecimentos pedagógicos dos conteúdos, buscando muitas vezes apresentar "metáforas" que exigem uma compreensão mais ampla. Assim, o desenvolvimento das atividades possibilitou aos futuros professores a reflexão sobre a própria prática, inserindo-se numa formação que vai ao encontro da concepção de Shulman (1986, 2005).

Nesse estudo, a discussão teórica e os resultados apresentados reforçam que a capacidade de um bom raciocínio visuoespacial tem particular significado no ensino e aprendizagem de geometria. Por isso, destaca-se a importância de futuros docentes explorarem uma educação enriquecida visuoespacialmente, pois também pode-se salientar que há fortes evidências de que as habilidades espaciais podem ser melhoradas com treinamento conforme os estudos de Newcombe e Stieff (2013). Do mesmo modo, práticas que estimulem as habilidades visuoespaciais dos seus alunos desde a educação básica, poderão proporcionar aumento no interesse profissional pelas áreas de matemática, ciências, engenharia e tecnologia (Uttal et al., 2013).

Por outro lado, a aprendizagem baseada em jogos digitais sérios tem sido discutida nos meios acadêmicos. Os jogos trazem elementos intrínsecos como a motivação e fazem parte da rotina de muitos acadêmicos, possibilitando construir coletivamente os conceitos necessários para a aprendizagem. Atividades nesse contexto expandem, diversificam e adaptam diversos ambientes de ensino e aprendizagem ao agregarem elementos como níveis, progressões e pontuações de forma lúdica, possibilitando maior engajamento dos alunos, favorecendo sua aprendizagem (Alves, 2014). 
Dessa forma, essa atividade não consistiu apenas na inserção de conteúdo educacional em um jogo de entretenimento, mas a adaptação do jogo de forma engajada com a aprendizagem, o que constituiu uma estratégia educacional com bons resultados, que levou o aprendiz a soluções criativas e ao uso das habilidades visuoespaciais, proporcionando atividades que poderão ser implantadas em sua atividade profissional. No entanto, ressalta-se a necessidade da integração de softwares de geometria dinâmica, como o Geogebra, entre outros, para continuação do ensino e aprendizagem e avaliação de geometria. Por fim, salienta-se que essa intervenção atendeu outro importante objetivo, que era possibilitar que futuros docentes transitem em experiências que envolvam o uso de tecnologias, exploração das habilidades visuoespaciais e raciocínio geométrico que possam vir a ser transferidas para a escola básica.

\section{Agradecimentos}

* Os autores agradecem ao fomento do Instituto Federal de Educação, Ciência e Tecnologia do Rio Grande do Sul - IFRS.

\section{Referências Bibliográficas}

ALSINA, C. C.; A FORTUNY, J. M.; PÉREZ, R. G. ¿Por qué geometría? propuestas didácticas para la ESO. Madrid: Síntesis, 1997.

ALVES, F. Gamification: como criar experiências de aprendizagem engajadoras - um guia completo: do conceito à prática. São Paulo: DVS, 2014.

BASSO, M. V. A.; NOTARE, M. Pensar-com tecnologias digitais de matemática dinâmica. RENOTE. Porto Alegre: CINTED-UFRGS, v.13, n.2, 2015.

BRASIL. Ministério da Educação. Conselho Nacional de Educação. Conselho Pleno. Resolução CNE/CP n. 02/2015. Brasília, 2015.

Secretaria de Educação Fundamental. Parâmetros Curriculares Nacionais: Matemática. Brasília: MEC/SEF, 1998. 148p

Secretaria de Educação Básica. Base Nacional Comum Curricular: Ensino Fundamental. Brasília: MEC/SEB, 2018a.

. Secretaria de Educação Básica. Base Nacional Comum Curricular: Ensino Médio (1 ${ }^{\mathrm{a}}$ versão). Brasília: MEC/SEB, 2018b.

BRUCE, C. D; HAWES, Z. The role of 2D and 3D mental rotation in mathematics for young children: what is it? Why does it matter? And what can we do about it? ZDM Mathematics Education, Berlin, v.47, n.3, p.331-343, 2015.

DAVIS, B.; SPATIAL REASONING STUDY GROUP. Spatial reasoning in the early years: principles, assertions, and speculations. New York: Routledge, 2015

EUCLIDEA. Faq. 2018. Disponível em: < https://www.euclidea.xyz/en/faq>. Acesso em: 17 abr. 2018.

GARTIC. Aprenda. 2016. Disponível em: < https://gartic.com.br/aprenda/>. Acesso em: 29 out. 2016.

GRAVINA, M. A. Geometria Dinâmica: uma nova abordagem para o aprendizado da geometria. In: SIMPÓSIO BRASILEIRO DE INFORMÁTICA NA EDUCAÇÃO, 6, 1996, Belo Horizonte. Anais. Belo Horizonte: SBIE, 1996. p.1-13.

HALLOWELL, D. A.; OKAMOTO, Y.; ROMO, L. F.; LA JOY, J. R. First-graders' spatialmathematical reasoning about plane and solid shapes and their representations. ZDM Mathematics Education, Berlin, v.47, n.3, p.363-375, 2015.

V. $16 \mathrm{~N}^{\mathrm{o}} 1$, julho, 2018 
KOSSLYN, S. M. Graph design for the eye and mind. New York: Oxford University Press, 2006.

LABORDE, C., KYNIGOS, C. HOLLEBRANDS, K., STRASSER, R. Teaching and learning geometry with technology. In: GUTIÉRREZ, A., BOERO, P. (eds.). Handbook of research on the psychology of mathematics education: past, present and future. Rotterdam: Sense Publishers, p. 275-304, 2006.

LOWRIE, T.; DIEZMANN, C. M. Solving graphics problems: student performance in the junior grades. The Journal of Educational Research, v.100, n.6, p.369-378, 2010.

LOWRIE, T.; LOGAN, T.; RAMFUL, A. Visuospatial training improves elementary students' mathematics performance. British Journal of Educational Psychology. Oxford, 87, p.170-186, 2017.

MOSS, J.; HAWES, Z. NAQVI, S.; CASWELL, B. Adapting Japanese Lesson Study to enhance the teaching and learning of geometry and spatial reasoning in early years classrooms: a case study. ZDM Mathematics Education, Berlin, v.47, n.3, p.377-390, 2015.

NATIONAL COUNCIL OF TEACHERS OF MATHEMATICS. NCTM. Principles and Standards for School Mathematics. United States: Reston, 2000. Disponível em: <www.nctm.org/standards>. Acesso em: 10 out. 2016

NEWCOMBE, N. S.; STIEFF, M. Six myths about spatial thinking. International Journal of Science Education, London, v.34, n.6, p.955-971, 2012.

OWENS, K. Diversifying our perspectives on mathematics about space and geometry: an ecocultural approach. International Journal of Science and Mathematics Education. Taiwan, v.12, n.4, p.941-974, 2014.

RAMFUL, A.; LOWRIE, T.; LOGAN, T. Measurement of spatial ability: construction and validation of the spatial reasoning instrument for middle school students. Journal of Psychoeducational Assessment. Califórnia, v. 35, n.7, p.709-727, 2017.

SCHON, D. A. Formar professores como profissionais reflexivos. In: NÓVOA, A. (Org.) Os professores e a sua formação. Lisboa: Dom Quixote, 1997. p.77 -91.

SHULMAN, L. S. Conocimiento y ensenãza: fundamentos de la nueva reforma. Profesorado. Revista de currículum y formación del profesorado, v.9, n.2, p.1-30, 2000.

. Those who understand: knowledge growth in teaching”. Educational Researcher, v.15, n.2, p.4-14, 1986.

SINCLAIR, N.; BRUCE, C. D. New opportunities in geometry education at the primary school. ZDM Mathematics Education, Berlin, v.47, n.3, p.319-329, 2015.

SINCLAIR, N.; BUSSI, M. G. B.; VILLIERS, M.; JONES, K.; KORTENKAMP, U.; LEUNG, A.; OWENS, K. Recent research on geometry education: an ICME-13 survey team report. ZDM Mathematics Education. Berlin, v.48, n.5, p.691-719, 2016.

SOURY-LAVERGNE, S.; MASCHIETTO, M. Articulation of spatial and geometrical knowledge in problem solving with technology at primary school. ZDM Mathematics Education, Berlin, v.47, n.3, p.435-449, 2015.

UTTAL, D. H.; MILLER, D. I.; NEWCOMBE, N. S. Exploring and enhancing spatial thinking: links to achievement in science, technology, engineering, and mathematics? Current Directions in Psychological Science, Califórnia, v. 22, n.5, p.367-373, 2013.

WAI, J.; LUBINSKI, D.; BENBOW, C. P. Spatial ability for STEM domains: aligning over 50 years of cumulative psychological knowledge solidifies its importance. Journal of Educational Psychology, Washington, v.101, n.4, p.817-835, 2009. 